\title{
3D EBSD Characterization of Deformed Polycrystalline Micro-scale Tensile Samples
}

\author{
M.A. Groeber*, P.A. Shade**, R. Wheeler*** and M.D. Uchic* \\ *Air Force Research Laboratory, Materials \& Manufacturing Directorate, AFRL/RXLMD, Wright \\ Patterson AFB, OH 45433 \\ ** Universal Technology Corporation, 1270 N Fairfield Road, Dayton, OH 45432 \\ *** UES, Inc., 4401 North Dayton-Xenia Rd, Dayton, OH 45432
}

This paper describes the application of a Dual Beam Focused Ion Beam-Scanning Electron Microscope (DB FIB-SEM) outfitted with an Electron Backscatter Diffraction (EBSD) System to characterize the internal microstructure and local lattice rotations within micro-scale test samples that have been deformed via in-situ SEM tensile testing. The objective of this study is to develop an experimental methodology that can provide (1) a high-fidelity $3 \mathrm{D}$ characterization of the internal grain structure of mechanical test samples that contain a limited number of grains (< 1000 grains) (2) knowledge of the external boundary conditions and measurement of the resultant stress-strain behavior of the same test samples, and (3) a 3D characterization of the internal lattice rotations that develop after a modest amount of plastic deformation $(\varepsilon \leq 5 \%)$. Such information could be used to assess and guide the further development of modeling and simulation methods that predict the local plastic deformation response of polycrystalline ensembles (i.e., 3D image-based modeling, see Refs. [1,2]).

An example of this experimental methodology is shown in Fig 1. The Ni-based superalloy micro-scale tension sample was fabricated via FIB milling, and was subsequently tested using a custom in-situ mechanical test system [3] to a total strain of $5.1 \%$, see Figs. 1A and 1B. Note that the displacement of the gage section was measured directly from SEM images that were collected continuously throughout the in-situ experiment. After testing, the sample was serial sectioned via FIB cross-section milling using a FEI Nova Nanolab microscope, where the average section thickness was approximately $125 \mathrm{~nm}$. An EBSD map was collected at each section [4] using a TSL OIM system with a Hikari EBSD detector, where the in-plane resolution of the crystallographic map matched the serial sectioning depth resulting in a cubic voxel for the reconstructed 3D data volume. In the associated presentation, we will discuss selected aspects of the experimental procedure, highlight methods used to post-process, segment, and classify grains from the serial sectioning data, and present an analysis of the grain microstructure and local lattice rotation data, as shown in Figs. 1C and 1D.

\section{References}

[1] G. Spanos et al., MRS Bulletin 33 (2008) 597-601.

[2] A.D. Rollett et al., in 'Proceedings of the International Conference on Microstructure and Texture in Steels.' (2009) Springer, London.

[3] P.A. Shade et al., Acta Materialia 57 (2009) 4580-4587.

[4] M.A. Groeber et al., Materials Characterization 57 (2006) 259-273. 

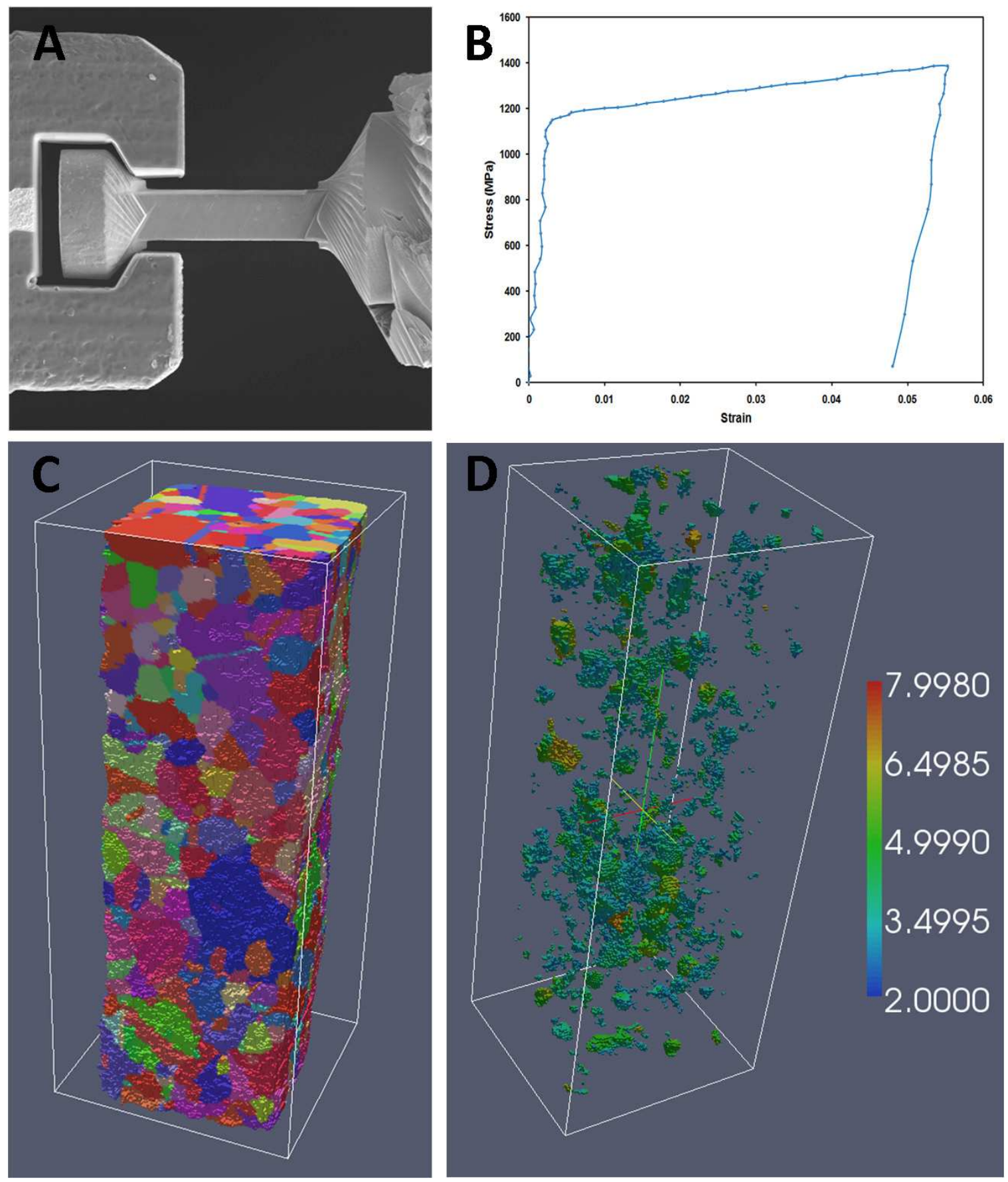

Figure 1. (A) SEM image of a polycrystalline LSHR Ni-based superalloy micro-tensile sample prior to testing. Dimensions of the parallelpiped sample gage section are $\sim 14 \mathrm{x}$ 14 x $45 \mu \mathrm{m}$. (B) Room temperature stress-strain response of the sample shown in Fig 1a. (C) Volume rendering of the grain structure within the gage section after deformation obtained by serial sectioning and EBSD mapping (D) Volume rendering of the local disorientation (determined relative to a reference orientation selected at the center of each grain), where the visible data represents disorientation values greater than 2 degrees. 